\title{
ADHD and EEG-neurofeedback: a double-blind randomized placebo-controlled feasibility study
}

\author{
M. M. Lansbergen • M. van Dongen-Boomsma • \\ J. K. Buitelaar · D. Slaats-Willemse
}

Received: 20 July 2010/ Accepted: 31 October 2010/Published online: 17 December 2010

(C) The Author(s) 2010. This article is published with open access at Springerlink.com

\begin{abstract}
Electroencephalography (EEG)-neurofeedback has been shown to offer therapeutic benefits to patients with attention-deficit/hyperactivity disorder (ADHD) in several, mostly uncontrolled studies. This pilot study is designed to test the feasibility and safety of using a doubleblind placebo feedback-controlled design and to explore the initial efficacy of individualized EEG-neurofeedback training in children with ADHD. Fourteen children (8-15 years) with ADHD defined according to the DSM-IV-TR criteria were randomly allocated to 30 sessions of EEG-neurofeedback $(n=8)$ or placebo feedback $(n=6)$. Safety measures (adverse events and sleep problems), ADHD symptoms and global improvement were monitored. With respect to feasibility, all children completed the study and attended all study visits and training sessions. No significant adverse effects or sleep problems were reported. Regarding the expectancy, $75 \%$ of children and their parent(s) in the active neurofeedback group and
\end{abstract}

Electronic supplementary material The online version of this article (doi:10.1007/s00702-010-0524-2) contains supplementary material, which is available to authorized users.

M. M. Lansbergen $(\bowtie) \cdot$ M. van Dongen-Boomsma .

D. Slaats-Willemse

Department of Psychiatry, Donders Institute for Brain, Cognition and Behaviour, Radboud University Nijmegen

Medical Centre, Nijmegen, The Netherlands

e-mail: mariekelansbergen@ @otmail.com

M. van Dongen-Boomsma · J. K. Buitelaar - D. Slaats-Willemse Karakter University Centre for Child and Adolescent Psychiatry, Nijmegen, The Netherlands

\section{J. K. Buitelaar}

Department of Cognitive Neuroscience,

Donders Institute for Brain, Cognition and Behaviour,

Radboud University Nijmegen, Nijmegen, The Netherlands
$50 \%$ of children and their parent(s) in the placebo feedback group thought they received placebo feedback training. Analyses revealed significant improvements of ADHD symptoms over time, but changes were similar for both groups. This pilot study shows that it is feasible to conduct a rigorous placebo-controlled trial to investigate the efficacy of neurofeedback training in children with ADHD. However, a double-blind design may not be feasible since using automatic adjusted reward thresholds may not work as effective as manually adjusted reward thresholds. Additionally, implementation of active learning strategies may be an important factor for the efficacy of EEGneurofeedback training. Based on the results of this pilot study, changes are made in the design of the ongoing study.

Keywords EEG-neurofeedback .

Attention-deficit/hyperactivity disorder (ADHD) .

Randomized controlled trial (RCT) .

Electroencephalogram (EEG) - Safety · Feasibility

\section{Introduction}

Attention-deficit/hyperactivity disorder (ADHD) is the most common psychiatric disorder in childhood, affecting about $5 \%$ of all children worldwide (Polanczyk et al. 2007). In 40-60\% of all cases ADHD persists in adolescence and adulthood, leading to a variety of problems such as poor academic performance, poor socialization, and increased traffic accidents (Faraone et al. 2006). Primary treatment for ADHD is medication, particularly psychostimulants. However, around $20 \%$ of all children with ADHD fail to respond to psychostimulants (Swanson et al. 1998) and in many responders there is still room for improvement. Moreover, minor and serious adverse side effects have been reported 
such as reduced growth, sleep disorders and decreased appetite (Charach et al. 2004, 2006). Long-term follow-up evaluation (22 months after the treatment period) has also indicated that a substantial part of the ADHD children that started medication at 7-9 years stopped within 2 years, after which clinical symptoms of ADHD reappeared (Jensen et al. 2007; Murray et al. 2008).

Electroencephalography (EEG)-neurofeedback appears to be a promising alternative or additional treatment without reported adverse effects (Arns et al. 2009; Gevensleben et al. 2009; Heinrich et al. 2007; Monastra et al. 2005). EEG-neurofeedback training involves the self-regulation of ongoing neuronal oscillations (recorded by EEG) in one or more frequency bands by visual or auditory feedback, aimed at normalizing and/or self-regulating brain activity. Changes made in the desired direction are rewarded, for example positively reinforced by presenting generally pleasant tones or pictures. Given the increased slow frequency oscillations (theta) and decreased high frequency oscillations (beta) in ADHD patients (Barry et al. 2003), one of the most often used and investigated EEG-neurofeedback training in ADHD involves increasing the production of beta activity $(16-20 \mathrm{~Hz})$ while suppressing the production of theta activity $(4-8 \mathrm{~Hz})$. The goal is to reduce symptoms of ADHD and to improve cognitive (and daily life) functioning (Gevensleben et al. 2009; Heinrich et al. 2007; Monastra et al. 2005). A different, frequently used EEG-neurofeedback training in ADHD involves suppressing of theta activity and simultaneously increasing sensorimotor rhythm (SMR; 12-15 Hz; Monastra et al. 2005). Neurofeedback training of slow cortical potentials (SCP) has also been conducted (Doehnert et al. 2008; Gevensleben et al. 2009), aimed at learning to regulate phasic (rather than tonic) cortical excitability. In SCP neurofeedback training, the task is to generate alternately negative and positive SCPs.

Seven controlled trials have assessed the efficacy of neurofeedback training (EEG and SCP) in children with ADHD, and demonstrated improvement of ADHD symptoms and cognitive functions (i.e., enhanced attention and inhibition) after neurofeedback training compared to a control condition (Drechsler et al. 2007; Gevensleben et al. 2009; Heinrich et al. 2004; Levesque et al. 2006) and similar improvements compared to psychostimulants (Fuchs et al. 2003; Monastra et al. 2002; Rossiter 2004). Moreover, recently a 6 months follow-up study provided evidence for long-term benefits of neurofeedback training in children with ADHD (Gevensleben et al. 2010). Changes in trained EEG oscillations after EEG-neurofeedback training are less straightforward and only demonstrated in two ADHD studies (Gevensleben et al. 2010; Monastra et al. 2002). Ambiguous changes in resting-state EEG activity after EEG-neurofeedback training have also been reported for other populations (e.g., Fernandez et al. 2007 in learning disabled children; Vernon 2005 in healthy volunteers), suggesting a reorganization of EEG activity rather than local changes in activity at trained electrode sites (Fernandez et al. 2007). A recent meta-analysis on the clinical effects of neurofeedback in ADHD reported large effect sizes for inattention and impulsivity and medium effects sizes for hyperactivity (Arns et al. 2009).

Although positive results after EEG-neurofeedback training in ADHD have been reported in seven controlled studies, it should be noted that the majority of studies had serious methodological problems. The most important methodological limitations of previous studies were small sample size, non-randomized group assignment, and control conditions that do not control for unspecific effects since they lack a double-blind setup (Heinrich et al. 2007).

Before EEG-neurofeedback treatment can be recommended as a standard treatment for ADHD and can be incorporated in current guidelines for ADHD, the results should be replicated and extended in more rigorous and scientifically controlled designs. Only in three studies, group assignment was randomized (Gevensleben et al. 2009; Heinrich et al. 2004; Levesque et al. 2006). The implementation of a control condition for EEG-neurofeedback is difficult: on the one hand it should be non-specific; on the other hand it should meet ethical standards (La Vaque and Rossiter 2001). Control conditions that have been used are a waiting list control group (Heinrich et al. 2004; Levesque et al. 2006), group therapy (Drechsler et al. 2007), and a computerized attention training (Gevensleben et al. 2009). Although group therapy and computerized attention training control for unspecific effects such as invested time and attention and treatment expectancies, from a methodological point of view the best control condition would be a placeboneurofeedback condition in which provided feedback is similar to neurofeedback, but not related to the child's own brain activity. The unspecific factors in this placebo-feedback condition are equal to the unspecific factors in an active EEG-neurofeedback training (e.g., Fernandez et al. 2007 in learning disabled children; Egner et al. 2002 in healthy volunteers; Angelakis et al. 2007 in elderly people; Logemann et al. 2010 in healthy volunteers). An important advantage of implementing placebo-feedback training is that it allows children and parents ${ }^{1}$ who participate in the study as well as researchers and trainers involved in the study to be blind for group allocation. This minimizes the effects of unspecific factors. However, implementing a placebo control condition in a randomized controlled trial may lead to ethical concerns when effective standard

\footnotetext{
${ }^{1}$ In the study from Gevensleben et al. (2009) parents were not informed about the treatment their child received and were not allowed into the room in which the intervention was given, but at the end of the training period approximately $60 \%$ of all parents could reliably indicate which training their child received.
} 
treatments like medication are available (La Vaque and Rossiter 2001). Moreover, it has been argued that placeboneurofeedback training is not feasible since it is clearly recognized by the patient (Kotchoubey et al. 2001).

In addition to a placebo-controlled design, it is important to monitor adverse events such as headaches and sleep problems, structurally during the training period to test the safety of (placebo)-neurofeedback training. None of the previous studies monitored potential adverse events.

So far, neurofeedback studies in ADHD children have used standardized neurofeedback training (mostly theta suppression/beta enhancement). Based on the observations that (1) not all children with ADHD have increased theta and decreased beta activity (Arns et al. 2008; Clarke et al. 2001; Van Dongen-Boomsma et al. 2010) and (2) increased theta in ADHD may partly be due to slow alpha oscillations rather than real enhanced theta activity (Lansbergen et al. 2010), EEG-neurofeedback training may be more effective when adjusted to the child's brain activity (i.e., individualized EEG-neurofeedback training). Indeed, this approach of individualized EEG-neurofeedback training is widely applied in clinical practice. The question remains whether individualized EEG-neurofeedback training as applied in clinical practice, may also be effective in improving symptoms of ADHD when applied in a scientific study design.

To summarize, EEG-neurofeedback training appears to be a promising treatment for ADHD, although a placebocontrolled double-blind trial including safety measures is needed to provide concluding evidence for the efficacy and safety of EEG-neurofeedback training in ADHD. Moreover, evidence for the efficacy of individualized EEGneurofeedback training (mostly applied in clinical practice) is lacking. In the present pilot study, the main objective is to test the feasibility and safety of using a rigorous doubleblind placebo feedback-controlled design in studying the effects of individualized neurofeedback training in children with ADHD. In order to provide a double-blind intervention, placebo/sham feedback was implemented which was equal to the EEG-neurofeedback training except that feedback was based on a simulated EEG signal instead of real brain activity (for similar approach, see Logemann et al. 2010). To meet the ethical standards, all participating children were allowed to continue their medication for ADHD, if any, and provided that there was enough room for further symptomatic improvement (see "Methods").

\section{Methods}

\section{Participants}

Children with ADHD (8-15 years) were recruited among referrals from Karakter University Centre for Child and
Adolescent Psychiatry Nijmegen and from parents who responded to advertisements. Children were included if (1) they had been diagnosed with ADHD as classified by the DSM-IV-TR (APA 2000), (2) they had an estimated IQ of at least 80, (3) their QEEG deviated at least 1.5 standard deviation (SD) from a normative database (see "EEGneurofeedback training and placebo feedback training"), and (4) they were psychopharmaca- naïve or -free, or used a stable dosage of psychostimulants or atomoxetine with room for improvement. Room for improvement was defined as an average score of more than $1 \mathrm{SD}$ above the mean on ADHD-DSM-IV rating scale (DuPaul et al. 1998). Children on a stable dosage of psychostimulants or atomoxetine were included in this study for several reasons. First, since the majority of ADHD children use medication, excluding them would preclude generalization of the results. Second, discontinuation of psychostimulants or atomoxetine in ADHD would be withholding an evidence-based and recommended treatment. Third, recent findings indicate that EEG-neurofeedback training should be embedded in a multimodal treatment program (Gevensleben et al. 2009).

Children were excluded if they (1) were involved in intensive (i.e., weekly) individual or group psychotherapy during the experiment, (2) used medication other than psycho-stimulants or atomoxetine, (3) had a comorbid disorder, other than oppositional defiant disorder (ODD) or an anxiety disorder, (4) had a neurological disorder and/or a cardiovascular disease, (5) participated in another clinical trial, (5) received neurofeedback training in the past, or (5) used alcohol or drugs.

So far (recruitment period: Sept 2008-April 2009), 26 parents and children (when older than 12 years of age) gave their written informed consent. Twelve children with ADHD had to be excluded from participation for the following reasons: 2 children did not show enough room for improvement, 2 children had an IQ below 80, 2 children did not have clear deviations in their EEG, 1 child had comorbid Gilles de la Tourette, 4 children did not have ADHD as main diagnosis and 1 child did not show up at the baseline measurements. Finally, 14 children with ADHD (mean age $10.2 \pm 2.0$ years; 1 girl) completed the study and were pseudo randomly double-blind allocated to one of the two groups. Eight children were assigned to the EEGneurofeedback training (10.4 \pm 2.3 years) and 6 children to the placebo feedback training $(10.0 \pm 1.7$ years; 1 girl $)$.

The study was approved by the Dutch Central Medical Ethics Committee (http://www.ccmo.nl) and conducted in accordance with the declaration of Helsinki. All parents and children older than 12 years of age gave their written informed consent before participation, children younger than 12 year gave their oral consent. Participation was rewarded by a present for the children. Travel expenses were partially reimbursed. 
Screening

First, all children were screened in a telephone interview with their parents addressing past and current ADHD symptoms; psychiatric and medical disorders, use of medication, intelligence level, and autistic traits (Social Communication Questionnaire, SCQ; Berument et al. 1999). A positive screening was followed by an extensive diagnostic procedure, including developmental interview, child psychiatric interview, and information by parents and teachers using scores on the Achenbach scales (CBCL and TRF), supervised by a child and adolescent psychiatrist. The diagnosis had to be confirmed by clinical scores on the ADHD-DSM-IV rating scale by the investigator (DuPaul et al. 1998). The presence of comorbid disorders was established in the child psychiatric interview and by using the Dutch electronic version of the Diagnostic Interview Schedule for Children (DISC-DSM-IV, parent version; Shaffer et al. 2000; Steenhuis et al. 2009). General functioning and severity of clinical symptoms were assessed using the Children's Global Assessment Scale (CGAS; Shaffer et al. 1983) and the Clinical Global ImpressionSeverity Scale (CGI-S; Bangs et al. 2008), respectively. If children's IQ was not assessed within the past 1.5 years, two subtests of the Wechsler Intelligence Scale for Children (WISC-III) were administered (i.e., Vocabulary and Block Design), to estimate intelligence. Validity coefficients for the Vocabulary and Block Design scores relative to the full form are 0.88 for verbal IQ and 0.83 for performance IQ (Antshel et al. 2007). Finally, a 20-min electroencephalogram (EEG) was recorded to assess whether children's QEEG deviated from the normative database (see "EEG-neurofeedback training and placebo feedback training").

\section{Study design}

A stratified, randomized, double-blind, sham-controlled between-subjects design was utilized. Children with ADHD were stratified according to age (i.e., younger vs. older than 12 years), use of medication (with vs. without medication), and EEG subtype (i.e., characterized by increased slow oscillations or by increased fast oscillations, as assessed by visual inspection of the raw EEG data), and subsequently randomly double-blind allocated to one of the following two groups: (1) EEG-neurofeedback and (2) placebo feedback.

All participating children, their parents, and all people involved in the study were blind to group assignment, except for the principal investigator who was not involved in data-collection, data-entry and data-analysis (J.K. Buitelaar).
EEG-neurofeedback training and placebo feedback training

Neurofeedback Instituut Nederland B.V. (NIN) provided EEG-neurofeedback training and placebo feedback training. To account for electrophysiological heterogeneity, individualized neurofeedback protocols were used for EEG-neurofeedback training. Individualized neurofeedback protocols were determined by NIN based on visual inspection of children's EEG recorded before training and based on the comparison of the quantitative EEG (QEEG) with the NeuroGuide database (http://www.applied neuroscience.com; Thatcher 1998) (Table 1, see also "Electrophysiological recordings"). The NeuroGuide database contains EEG data from 625 healthy individuals (58.9\% males) of which 470 are children between 1 and 15 years old. For all subjects in the database, EEG data was recorded from 19 electrodes placed according to the 10-20 electrode international system and referenced to linked ear lobes during an eyes closed and eyes open resting-state condition (Thatcher 1998).

For all children in the present study, eyes open and eyes closed raw EEG data were visually inspected and quantitative EEG (QEEG) data from each participant before EEG-neurofeedback training was analyzed using Deymed Truescan software. EEG data with eye movements and artifacts were removed. Subsequently, individual QEEGs were compared to the NeuroGuide database and deviations from the normative database were identified (FFT maximal $z$ scores). The aim of the EEG-neurofeedback training was to normalize power within specific frequency bands and at specific electrode sites (for the same procedure, see Logemann et al. 2010). As shown in Table 1, most participants were learned to increase the production of sensorimotor rhythm (SMR) while simultaneously suppressing the production of theta activity.

BrainMaster Atlantis hardware and software was used to provide EEG-neurofeedback training and placebo-feedback training. Identical procedures were provided to the children

Table 1 Individualized EEG-neurofeedback training protocols

\begin{tabular}{lllll}
\hline Child & Site & $\begin{array}{l}\text { Theta stop } \\
(\mathrm{Hz})\end{array}$ & $\begin{array}{l}\text { SMR go } \\
(\mathrm{Hz})\end{array}$ & $\begin{array}{l}\text { High stop } \\
(\mathrm{Hz})\end{array}$ \\
\hline 997 & F3 and F4 & $4-7$ & $12-15$ & $20-30$ \\
999 & F3 and F4 & $4-7$ & $12-15$ & $20-30$ \\
995 & C3 and C4 & $4-7$ & $12-15$ & \\
992 & P3 and C4 & $4-6$ & $12-15$ & \\
989 & P3 and P4 & $4-7$ & $12-15$ & \\
987 & Fz & $4-7$ & $12-15$ & \\
984 & C3 and C4 & $4-7$ & $12-15$ & \\
983 & C3 and C4 & & & $15-20+20-25$
\end{tabular}


in the EEG-neurofeedback group and placebo feedback group, except that children in the EEG-neurofeedback group received feedback on their real-time EEG-signal, whereas children in the placebo feedback group received feedback on a simulated EEG signal, generated by BrainMaster Atlantis software (Logemann et al. 2010). The simulated EEG signal consisted of a random signal similar to real EEG. Feedback to a real EEG signal and the simulated EEG signal were similar in experience (Logemann et al. 2010). During training, all children watched a movie for $20 \mathrm{~min}$. They were asked to sit as quiet as possible in a comfortable arm chair in front of a 17-19 in. TFT computer screen showing a part of the movie. EEG data were obtained from the active electrode(s) placed on the scalp at the location(s) of interest (Table 1). The reference was linked ears or left ear if the active electrode was placed on the middle of the scalp. All electrode impedances were kept under $6 \mathrm{k} \Omega$. Sampling rate was $256 \mathrm{~Hz}$. EEG data were filtered (DC-120 Hz) and de-artefacted online (peakto-peak amplitude criterion of $120 \mu \mathrm{V}$ ). Positive feedback was provided by both brightening the computer screen and presenting an auditory tone when the production of SMR (estimated from the filtered and de-artefacted EEG signal) remained above threshold, and/or theta and beta activity (estimated from the filtered and de-artefacted EEG signal) remained below threshold. Reward threshold levels were automatically adjusted based on the digitally filtered realtime EEG signal every 30 s so that the child was rewarded about $80 \%$ of the time (i.e., received positive feedback). Consequently, the amount of reward remained at about the same level across sessions and across groups. During training, children were instructed to try to self-regulate their brain activity by receiving positive feedback based on the real-time EEG signal. The children were trained over a period of approximately 4 months with 2 sessions per week, in total 30 sessions. The duration of each session was $45 \mathrm{~min}$ and included approximately $20 \mathrm{~min}$ of uninterrupted EEG-neurofeedback or placebo feedback training. Training was conducted in an 'active focusing state' with eyes open.

\section{Outcome measures}

In the present paper, the feasibility of the study design, the safety results and the results regarding the effects of EEGneurofeedback training in comparison with placebo feedback training on ADHD symptom severity and global improvement, rated by the investigator, are reported and discussed. The results regarding the effects of EEGneurofeedback training on trained EEG oscillations are provided in the electronic supplementary material. Unfortunately, EEG data during training sessions were not recorded. Cognitive data for the whole group and magnetic resonance images (MRI) data acquired for a subgroup of children are not reported here.

Feasibility measures

Feasibility was assessed by attendance of the study visits and the training sessions. Additionally, in the final interview with the investigator after the treatment period, parents and children were asked to indicate whether they thought the child had received EEG-neurofeedback training or placebo feedback training.

\section{Safety measures}

To evaluate the safety, i.e., potential adverse effects of EEG-neurofeedback or placebo-feedback training, parents and children filled out the Pittsburgh side effects rating scale (PSERS; Pelham et al. 2001; Sandler and Bodfish 2008) before training and after 6, 10, 20, and 30 training sessions. The PSERS measures the presence/absence and severity ( $1=$ mild, $2=$ moderate, $3=$ severe $)$ of several potential side effects (e.g., tics, skin picking, sleepiness, headache, stomach ache, irritability, and appetite loss). For the present study, three items were added to the original PSERS: epileptic seizures, nausea, and feeling agitated. Severity of adverse effects was calculated as the sum score of all 15 items. For 1 child from the EEG-neurofeedback group, adverse events after the training period were not rated.

Sleep problems were assessed using 14 insomnia items of the Dutch version of the Sleep Disorders Questionnaire (SDQ; Sweere et al. 1998) before and after the treatment period. Parents and children rated each item on a five-point rating scale $(0=$ never, $1=$ rarely, $2=$ sometimes, 3 = usually, 4 = always) before and after the training period. Total severity of sleep problems was defined as the sum score of all items. For 1 child from the EEG-neurofeedback group, sleep problems were not rated after the training period.

\section{Efficacy measures}

Efficacy in the present pilot study was measured by the total severity of inattention and hyperactive/impulsive symptoms of ADHD according to the ADHD DSM-IV scale (DuPaul et al. 1998), rated by the investigator in an interview with the parents. Severity of the ADHD symptoms were rated (with a score from 0 to 3 ) before training, after 6, 10, 20, 30 training sessions, and 6 months after the end of the training period.

Global improvement was also included as a measure of efficacy and was assessed in the final interview with the parents using the Clinical Global Impressions-Improvement 
scale (CGI-I; Wigal et al. 2006). The CGI-I is a widely used scale to evaluate clinical effects in intervention studies. CGII consists of a single item 7 -point $(1=$ very much improved, $2=$ much improved, $3=$ minimally improved, $4=$ no change, $5=$ minimally worse, $6=$ much worse, $7=$ very much worse). CGI-I responders were defined as children that were rated as very much improved or much improved. The CGI-I scale was missing for one child in the EEG-neurofeedback training group.

\section{Electrophysiological recordings}

Before and after training, EEG was recorded using DeyMed TrueScan and an Electrocap with 19 electrodes (according to the 10-20 electrode international system) during a 10-min eyes open and 10-min eyes closed resting-state condition. Data were referenced to a common reference placed between Fpz and Fz. The ground electrode was placed at the forehead. EEG data were filtered online with a bandwidth of $0.1-102 \mathrm{~Hz}$. Sampling rate was $256 \mathrm{~Hz}$. Electrode impedance was kept below $10 \mathrm{k} \Omega$.

Children were instructed to sit quietly for $20 \mathrm{~min}$, $10 \mathrm{~min}$ with eyes open and $10 \mathrm{~min}$ with eyes closed.

\section{Study procedure}

After recruitment, screening and baseline assessments, EEG-neurofeedback training or placebo feedback training started for a period of approximately 4-5 months. After 6 training sessions, DSM-IV TR ADHD criteria were evaluated in a telephone interview with the parents. Additionally, parents filled out the PSERS to rate any adverse events. After 10 and 20 training sessions, DSM-IV TR ADHD criteria were again evaluated in a telephone interview with the parents, and parents filled out the PSERS. After the training period of 30 training sessions, DSM-IV TR ADHD criteria were again evaluated in a telephone interview with the parents, and parents filled out the PSERS and SDQ. Furthermore, the EEG recordings, neuropsychological test battery and MRI session were repeated. In a final interview by the investigator with the parents, general clinical improvement (CGI-I) was assessed. Additionally, the experiences of the training were discussed and parents and children were asked to indicate whether they thought the child had received EEG-neurofeedback training or placebo feedback training. Six months after the end of the training period, DSM-IV TR ADHD criteria were evaluated in a telephone interview with the parents.

Since the main objective of the present paper was to evaluate the feasibility and safety of using a placebo-controlled design to test the efficacy of EEG-neurofeedback training in children with ADHD, the present paper focuses on the feasibility and safety measures and some initial results with respect to ADHD symptoms and global improvement.

\section{Statistical analysis}

We conducted repeated-measures analysis of variance (ANOVA) (two-tailed, significance level is set at 5\%) with TIME as within-subjects factor and GROUP (EEG-neurofeedback vs. placebo neurofeedback) as between-subjects factor separately for the severity of adverse events, severity of sleep problems (SDQ), severity of inattention symptoms, and severity of hyperactive/impulsive symptoms. For severity of adverse events and the severity of inattention symptoms and hyperactive/impulsive symptoms, withinsubject factor TIME had 5 levels (i.e., before and after 6 , 10, 20 and 30 training sessions). For the severity of sleep problems (SDQ), TIME had 2 levels (before and after training).

Follow-up assessment of the severity of ADHD symptoms, rated by the investigator, was compared with the severity of ADHD symptoms immediately after the training period using repeated-measures ANCOVAs with between-subject factor GROUP (EEG-neurofeedback vs. placebo neurofeedback), within-subject factor TIME (posttraining, follow-up) using the baseline (pre-training) measure as a covariate.

\section{Results}

At baseline, all children were rated as moderately ill ( $n=11)$ or markedly ill $(n=3)$ by the psychiatrist using the CGI-S. Baseline assessments of ADHD severity, based on the ADHD DSM-IV scale, indicated enough room for improvement for all children (Table 2). Five of 8 children in the EEG-neurofeedback group and 4 of 6 children in the placebo feedback group were medicated with psychostimulants (methylphenidate or dextroamphetamine; for details, see Table 2). None of the children changed type or dose of medication during participation of the study.

\section{Feasibility}

From the 14 children with ADHD who started the EEGneurofeedback or placebo feedback training, all children completed the study. Additionally, participating children and parents attended all study visits and all training sessions. Regarding the expectancy of group assignment, 2 of 8 children and their parent(s) in the active EEG-neurofeedback group and 2 of 6 children and their parent(s) in the placebo feedback group thought they received active 
Table 2 Dosage and type of medication, and ADHD severity at baseline (based on the ADHD DSM-IV scale) for each child

\begin{tabular}{lllll}
\hline Child & Group & Medication & \multicolumn{2}{l}{ ADHD severity-baseline } \\
\cline { 4 - 5 } & & & Inattention & Hyp/Imp \\
\hline 999 & NF & MPH $(5 \mathrm{mg})+$ concerta $(54 \mathrm{mg})$ & 17 & 11 \\
997 & NF & MPH $(15-10-5 \mathrm{mg})$ & 5 & 20 \\
995 & NF & MPH $(10 \mathrm{mg})+$ concerta $(36 \mathrm{mg})$ & 16 & 18 \\
992 & NF & Concerta $(36 \mathrm{mg})+$ melatonine $(3 \mathrm{mg})$ & 21 & 23 \\
989 & NF & - & 23 & 17 \\
987 & NF & - & 26 & 17 \\
984 & NF & - & 26 & 1 \\
983 & NF & MPH $(10-10-5 \mathrm{mg})+$ melatonine $(2.5 \mathrm{mg})$ & 18 & 15 \\
998 & Placebo & MPH $(10-10 \mathrm{mg})$ & 21 & 26 \\
996 & Placebo & - & 12 & 14 \\
994 & Placebo & MPH $(10-10 \mathrm{mg})+$ melatonine $(3 \mathrm{mg})$ & 24 & 10 \\
993 & Placebo & MPH $(10 \mathrm{mg})+$ concerta $(18 \mathrm{mg})$ & 17 & 21 \\
990 & Placebo & d-amf $(7.5-7.5 \mathrm{mg})+$ melatonine $(3 \mathrm{mg})$ & 23 & 16 \\
986 & Placebo & - & 18 & 20 \\
\hline
\end{tabular}

EEG-neurofeedback training. Another child in the placebo feedback group thought he received active EEG-neurofeedback, whereas his parents thought he received placebo feedback. The other children and their parent(s) [6 of 8 $(75 \%)$ in the active EEG-neurofeedback group; 3 of 6 $(50 \%)$ in the placebo feedback group] thought the child received placebo feedback training. So, parent's and child's prediction of which training group the child was assigned to was at chance level.

\section{Safety}

The average number of 'adverse events' before the start of the EEG-neurofeedback training was $2.5 \pm 2.4$ (with a total severity of $3.9 \pm 3.8$ ). "Skin picking" and "irritable" were the most often reported adverse events. For all children, the severity of adverse events did not increase or decrease over time during the training period (Table 3). Total severity of sleep problems did also not significantly increase or decrease over time (Table 3).

\section{Efficacy}

Table 3 presents behavioral data before and after training, separately for the EEG-neurofeedback and placebo feedback training group. As shown in Figs. 1a and 1b, the total severity of DSM-IV symptoms of inattention and hyperactivity/impulsivity rated by the investigator decreased significantly over time (main effect of time: $F_{(4,48)}=$ 22.07, $p<.001$ and $F_{(4,48)}=8.09, p<.001$ for inattention and hyperactivity/impulsiveness, respectively) but the improvement in ADHD symptoms were present in both groups (i.e., there was no significant time $\times$ group interaction effects).
Table 3 Behavioral data before training and after training for the EEG-neurofeedback group and placebo-feedback group

\begin{tabular}{lrrrrr}
\hline & \multicolumn{2}{l}{ EEG-neurofeedback } & & \multicolumn{2}{l}{ Placebo-feedback } \\
\cline { 2 - 3 } & \multicolumn{1}{c}{ Pre } & \multicolumn{1}{c}{ Post } & & Pre & \multicolumn{1}{c}{ Post } \\
\hline Severity sleep problems & $13.7(7.3)$ & $9.3(4.7)$ & & $9.5(8.0)$ & $8.5(7.1)$ \\
Severity adverse events & $3.3(3.8)$ & $2.3(1.7)$ & & $3.8(3.8)$ & $3.0(3.9)$ \\
Severity Inatt-parent & $19.0(6.8)$ & $13.4(7.8)$ & & $19.2(4.4)$ & $12.5(2.3)$ \\
Severity Hyp/Imp-parent & $15.3(6.7)$ & $10.3(6.0)$ & & $17.8(5.7)$ & $14.7(6.2)$ \\
CGI-I & & $3.4(0.8)$ & & $3.7(0.5)$
\end{tabular}

Reduced scores reflect improvement for all scales. Clinical Global Impression (CGI) score ranged from 1 (very much improved) to 7 (very much worse). Standard deviations of the mean are given in parentheses

Inatt DSM-IV inattentive symptoms, Hyp/Imp DSM-IV hyperactive/ impulsive symptoms, CGI-I Clinical Global Impression-Improvement

CGI-I results showed that only one child (in the EEGneurofeedback training group) was rated as a responder (i.e., rated as "much improved"). Two children in the EEG-neurofeedback group and 2 children in the placebo feedback group were rated as "minimal improved". Four of 8 children in the EEG-neurofeedback training group and 4 of 6 children in the placebo feedback training group did not show any clinical global improvements. None of the children deteriorated.

Regarding the follow-up assessment of total severity of DSM-IV symptoms of inattention and hyperactivity/ impulsivity, repeated measures ANCOVA did not yield significant main effects of group or time, or a significant group $\times$ time interaction effect. These results indicate no differences between ADHD symptoms directly after the training period and ADHD symptoms 6 months after the end of the training period. 

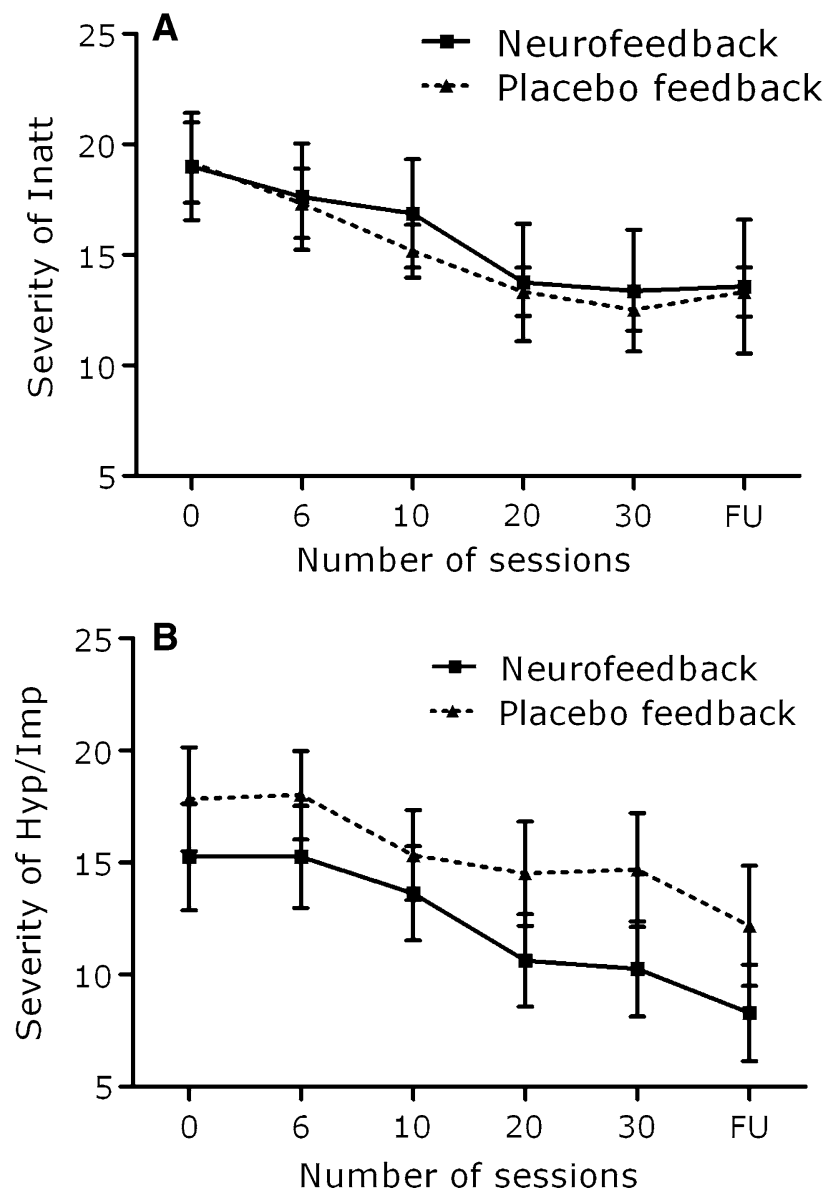

Fig. 1 Total severity of ADHD Inattentive symptoms (a) and ADHD hyperactive/impulsive symptoms (b) before training and after 6, 10, 20, 30 sessions of EEG-neurofeedback, and at follow-up (FU; 6 months) for the EEG-neurofeedback and placebo-feedback group

\section{Discussion}

Consistent evidence for improvements in ADHD symptoms after EEG-neurofeedback training in children with ADHD have been provided by several studies (Arns et al. 2009; Gevensleben et al. 2009; Heinrich et al. 2007). However, the majority of these studies have methodological limitations. A rigorous double-blind placebo-controlled trial has not yet been published in a peer-reviewed international journal. The main reasons for the lack of such a complementary design are (1) the ethical issue of withholding evidence-based treatment and (2) the doubts about the feasibility of placebo-neurofeedback (Kotchoubey et al. 2001; La Vaque and Rossiter 2001). The main objective of the present pilot study was to test the feasibility and safety of using a rigorous double-blind placebo feedback-controlled design in studying the effects of individualized neurofeedback training in children with ADHD. To overcome the problem of withholding evidencebased treatment, children were allowed to use medication, but were not allowed to switch drug or dosage.
To account for electrophysiological heterogeneity, individualized neurofeedback protocols were determined for the participating children based on their baseline EEG activity. As presented in Table 1, most children received theta suppression/SMR enhancement training, which is one of the most often used neurofeedback training protocols (Monastra et al. 2005).

In this pilot study, all included children completed the 30 training sessions and attended all study visits. With respect to safety, neither EEG-neurofeedback training nor placebo-feedback training evoked significant adverse events or sleep problems, indicating that EEG-neurofeedback and placebo feedback may not have serious side effects. Further, 3 of 6 children from the placebo-feedback group thought they received active EEG-neurofeedback training. Providing placebo neurofeedback training as a control condition thus appears to be a 'feasible' approach. Indeed, placebo feedback has previously been applied in other populations (learning disabled children, Fernandez et al. 2007; elderly, Angelakis et al. 2007; healthy volunteers, Egner et al. 2002; Logemann et al. 2010).

Blinded analysis of this ongoing EEG-neurofeedback study demonstrated clinical improvement over time, as reflected in reduced ADHD DSM-IV symptoms rated by the investigator, but did not reveal significant differences between the EEG-neurofeedback training group and placebo feedback group. Thus, individualized EEG-neurofeedback training did not outperform placebo feedback training in improvement of clinical symptoms in ADHD children. Of course, the power of this interim sample is limited, as we had projected to need a sample of 120 subjects in total. However, not even a trend was found for any group $\times$ time interaction effects $(F<1)$. The present findings suggest that the behavioral improvements observed in this study after individualized EEG-neurofeedback training may not be caused by the ability to selfregulate brain activity, but rather by unspecific effects such as invested time and attention, therapist interaction, expectancy, or just by passed time. This finding may raise doubt on the positive results of previous studies that did not completely control for unspecific factors. However, several important issues have to be discussed. First, the EEGneurofeedback training as provided in the present study was adjusted to each child (i.e., individualized EEGneurofeedback), whereas previous studies all provided standardized neurofeedback training. It may be speculated that individualized EEG-neurofeedback as mostly applied in practice with the aim to normalize 'deviant' brain activity is not effective, whereas standardized EEG-neurofeedback training with the aim to self-control brain activity is effective. The deviation from the normative EEG database may just indicate electrophysiological heterogeneity that is normal or even adaptive rather than pathological. Second, 
to realize a double-blind design in which even the EEGneurofeedback therapist did not know to which group the child was assigned, automatic reward threshold adjustments were used to provide EEG-neurofeedback training. However, EEG-neurofeedback training based on automatic adjusted reward thresholds might not work as effective as EEG-neurofeedback training based on manual adjusted reward thresholds (Logemann et al. 2010). In line with this suggestion, the majority of children and their parents from the active EEG-neurofeedback training group (6 of 8) thought they received placebo feedback training. A third limitation of this study is the lack of actively practicing mental strategies to self-regulate brain activity (e.g., Gevensleben et al. 2009). In other words, EEG-neurofeedback might need explicit learning rather than implicit learning. Finally, as indicated by Monastra et al. (2005), it may be important to control for variations in parenting style that may mediate treatment response.

Given these limitations and in the first place the small sample size in this study, we believe it is preliminary to conclude that individualized EEG-neurofeedback training is not effective in improving ADHD symptoms. We may conclude from the present findings that it is feasible to conduct a rigorous placebo-controlled trial to investigate the efficacy of EEG-neurofeedback training in children with ADHD placebo-feedback, arguing against earlier statements that placebo feedback is impossible (Kotchoubey et al. 2001). However, a double-blind design may not be feasible since using automatic adjusted reward thresholds may not work as effective as manually adjusted reward thresholds.

Based on these results we have made two changes in our ongoing EEG-neurofeedback study. First, we will provide EEG-neurofeedback training in which the trainer adjusts manually the feedback parameters with the consequence that the trainer will not be blind to group assignment. Children, their parents, and all other people involved in the study will be still blind to group assignment. Second, we will assist children in developing and practicing active learning strategies to self-regulate brain activity (e.g., focusing one's attention) and promote the children to implement the acquired strategies in daily-life situations, aimed at optimizing the therapeutic effects of EEG-neurofeedback training (see Gevensleben et al. 2009).

Acknowledgments The authors gratefully acknowledge the support of the BrainGain Smart Mix Programme of The Netherlands Ministry of Economic Affairs and The Netherlands Ministry of Education, Culture and Science. We also thank Alexander Logemann who made it possible to give double-blind EEG-neurofeedback training; Dorith Merkx, Andrieke Thissen and Madelon Vollebregt for their help in testing the children; Martijn Arns for his advice in EEG-neurofeedback training; Neurofeedback Instituut Netherlands (Ger Loots, Derk Mulder, and the EEG-neurofeedback trainers) for providing the EEGneurofeedback training; Nadine Schalk who was responsible for the logistics; and finally all children and parents who were willing to participate in this extensive study.

Conflict of interest Professor Buitelaar has been employed as a consultant and participated in advisory board meetings and speakers bureau for Eli Lilly, Janssen-Cilag, USB, Shire, Medice, BristolMyers Squibb and Pfizer. All other authors declare that they have no conflicts of interest.

Open Access This article is distributed under the terms of the Creative Commons Attribution Noncommercial License which permits any noncommercial use, distribution, and reproduction in any medium, provided the original author(s) and source are credited.

\section{References}

American Psychiatric Association (2000) Diagnostic and statistical manual of mental disorders, 4th edn. text revision (DSM-IVTR). American Psychiatric Association Press, Washington (DC)

Angelakis E, Stathopoulou S, Frymiare JL, Green DL, Lubar JF, Kounios J (2007) EEG neurofeedback: a brief overview and an example of peak alpha frequency training for cognitive enhancement in the elderly. Clin Neuropsychol 21:110-129

Antshel KM, Faraone SV, Stallone K, Nave A, Kaufmann FA, Doyle A et al (2007) Is attention deficit hyperactivity disorder a valid diagnosis in the presence of high IQ? Results from the MGH longitudinal family studies of ADHD. J Child Psychol Psychiatry 48:687-694

Arns M, Gunkelman J, Breteler M, Spronk D (2008) EEG phenotypes predict treatment outcome to stimulants in children with ADHD. J Integr Neurosci 7:421-438

Arns M, de Ridder S, Strehl U, Breteler M, Coenen A (2009) Efficacy of neurofeedback treatment in ADHD: the effects on inattention, impulsivity and hyperactivity: a meta-analysis. Clin EEG Neurosci 40:180-189

Bangs ME, Hazell P, Danckaerts M, Hoare P, Coghill DR, Wehmeier PM et al (2008) Atomoxetine for the treatment of attentiondeficit/hyperactivity disorder and oppositional defiant disorder. Pediatrics 121:e314-e320

Barry RJ, Clarke AR, Johnstone SJ (2003) A review of electrophysiology in attention-deficit/hyperactivity disorder: I. Qualitative and quantitative electroencephalography. Clin Neurophysiol 114:171-183

Berument SK, Rutter M, Lord C, Pickles A, Bailey A (1999) Autism screening questionnaire: diagnostic validity. $\mathrm{Br} \mathrm{J}$ Psychiatry 175:444-451

Charach A, Ickowicz A, Schachar R (2004) Stimulant treatment over five years: adherence, effectiveness, and adverse effects. J Am Acad Child Adolesc Psychiatry 43:559-567

Charach A, Figueroa M, Chen S, Ickowicz A, Schachar R (2006) Stimulant treatment over 5 years: effects on growth. J Am Acad Child Adolesc Psychiatry 45:415-421

Clarke AR, Barry RJ, McCarthy R, Selikowitz M (2001) Excess beta activity in children with attention-deficit/hyperactivity disorder: an atypical electrophysiological group. Psychiatry Res 103:205-218

Doehnert M, Brandeis D, Straub M, Steinhausen HC, Drechsler R (2008) Slow cortical potential neurofeedback in attention deficit hyperactivity disorder: is there neurophysiological evidence for specific effects? J Neural Transm 115:1445-1456

Drechsler R, Straub M, Doehnert M, Heinrich H, Steinhausen HC, Brandeis D (2007) Controlled evaluation of a neurofeedback training of slow cortical potentials in children with Attention deficit/hyperactivity disorder (ADHD). Behav Brain Funct 3:35 
DuPaul GJ, Power TJ, Anastopoulos AD, Reid R (1998) ADHD Rating Scale-IV. Checklists, norms and clinical interpretations. Guilford Press, New York

Egner T, Strawson E, Gruzelier JH (2002) EEG signature and phenomenology of alpha/theta neurofeedback training versus mock feedback. Appl Psychophysiol Biofeedback 27:261-270

Faraone SV, Biederman J, Mick E (2006) The age-dependent decline of attention deficit hyperactivity disorder: a meta-analysis of follow-up studies. Psychol Med 36:159-165

Fernandez T, Harmony T, Fernandez-Bouzas A, az-Comas L, PradoAlcala RA, Valdes-Sosa P et al (2007) Changes in EEG current sources induced by neurofeedback in learning disabled children. An exploratory study. Appl Psychophysiol Biofeedback 32:169-183

Fuchs T, Birbaumer N, Lutzenberger W, Gruzelier JH, Kaiser J (2003) Neurofeedback treatment for attention-deficit/hyperactivity disorder in children: a comparison with methylphenidate. Appl Psychophysiol Biofeedback 28:1-12

Gevensleben H, Holl B, Albrecht B, Vogel C, Schlamp D, Kratz O et al (2009) Is neurofeedback an efficacious treatment for ADHD? A randomised controlled clinical trial. J Child Psychol Psychiatry 50:780-789

Gevensleben H, Holl B, Albrecht B, Schlamp D, Kratz O, Studer P et al (2010) Neurofeedback training in children with ADHD: 6-month follow-up of a randomised controlled trial. Eur Child Adolesc Psychiatry 19:715-724

Heinrich H, Gevensleben H, Freisleder FJ, Moll GH, Rothenberger A (2004) Training of slow cortical potentials in attention-deficit/ hyperactivity disorder: evidence for positive behavioral and neurophysiological effects. Biol Psychiatry 55:772-775

Heinrich H, Gevensleben H, Strehl U (2007) Annotation: neurofeedback - train your brain to train behaviour. J Child Psychol Psychiatry 48:3-16

Jensen PS, Arnold LE, Swanson JM, Vitiello B, Abikoff HB, Greenhill LL et al (2007) 3-year follow-up of the NIMH MTA study. J Am Acad Child Adolesc Psychiatry 46:989-1002

Kotchoubey B, Strehl U, Uhlmann C, Holzapfel S, Konig M, Froscher $\mathrm{W}$ et al (2001) Modification of slow cortical potentials in patients with refractory epilepsy: a controlled outcome study. Epilepsia 42:406-416

La Vaque TJ, Rossiter T (2001) The ethical use of placebo controls in clinical research: the Declaration of Helsinki. Appl Psychophysiol Biofeedback 26:23-37

Lansbergen MM, Arns M, Van Dongen-Boomsma M, Spronk D, Buitelaar JK (2010) The increase in theta/beta ratio on restingstate EEG in boys with attention-deficit/hyperactivity disorder is mediated by slow alpha peak frequency. Prog Neuropsychopharmacol Biol Psychiatry. doi:10.1016/j.pnpbp.2010.08.004

Levesque J, Beauregard M, Mensour B (2006) Effect of neurofeedback training on the neural substrates of selective attention in children with attention-deficit/hyperactivity disorder: a functional magnetic resonance imaging study. Neurosci Lett 394:216-221

Logemann HN, Lansbergen MM, Van Os TW, Bocker KB, Kenemans JL (2010) The effectiveness of EEG-feedback on attention, impulsivity and EEG: a sham feedback controlled study. Neurosci Lett 479:49-53

Monastra VJ, Monastra DM, George S (2002) The effects of stimulant therapy, EEG biofeedback, and parenting style on the primary symptoms of attention-deficit/hyperactivity disorder. Appl Psychophysiol Biofeedback 27:231-249

Monastra VJ, Lynn S, Linden M, Lubar JF, Gruzelier J, LaVaque TJ (2005) Electroencephalographic biofeedback in the treatment of attention-deficit/hyperactivity disorder. Appl Psychophysiol Biofeedback 30:95-114

Murray DW, Arnold LE, Swanson J, Wells K, Burns K, Jensen P et al (2008) A clinical review of outcomes of the multimodal treatment study of children with attention-deficit/hyperactivity disorder (MTA). Curr Psychiatry Rep 10:424-431

Pelham WE, Gnagy EM, Burrows-Maclean L, Williams A, Fabiano GA et al (2001) Once-a-day Concerta methylphenidate versus three-times-daily methylphenidate in laboratory and natural settings. Pediatrics 107:E105

Polanczyk G, de Lima MS, Horta BL, Biederman J, Rohde LA (2007) The worldwide prevalence of ADHD: a systematic review and metaregression analysis. Am J Psychiatry 164:942-948

Rossiter T (2004) The effectiveness of neurofeedback and stimulant drugs in treating AD/HD: part II. Replication. Appl Psychophysiol Biofeedback 29:233-243

Sandler AD, Bodfish JW (2008) Open-label use of placebos in the treatment of ADHD: a pilot study. Child Care Health Dev 34:104-110

Shaffer D, Gould MS, Brasic J, Ambrosini P, Fisher P, Bird H, Aluwahlia S (1983) A children's global assessment scale (CGAS). Arch Gen Psychiatry 40:1228-1231

Shaffer D, Fisher P, Lucas CP, Dulcan MK, Schwab-Stone ME (2000) NIMH Diagnostic Interview Schedule for Children Version IV (NIMH DISC-IV): description, differences from previous versions, and reliability of some common diagnoses. J Am Acad Child Adolesc Psychiatry 39:28-38

Steenhuis MP, Serra M, Minderaa RB, Hartman CA (2009) An Internet version of the Diagnostic Interview Schedule for Children (DISC-IV): correspondence of the ADHD section with the paper-and-pencil version. Psychol Assess 21:231-234

Swanson JM, Sergeant JA, Taylor E, Sonuga-Barke EJ, Jensen PS, Cantwell DP (1998) Attention-deficit hyperactivity disorder and hyperkinetic disorder. Lancet 351:429-433

Sweere Y, Kerkhof GA, De Weerd AW, Kamphuisen HA, Kemp B, Schimsheimer RJ (1998) The validity of the Dutch Sleep Disorders Questionnaire (SDQ). J Psychosom Res 45:549-555

Thatcher RW (1998) EEG normative databases and EEG biofeedback. J Neurotherapy 2:8-39

Van Dongen-Boomsma M, Lansbergen MM, Bekker EM, Kooij JJ, van der MM, Kenemans JL, Buitelaar JK (2010) Relation between resting EEG to cognitive performance and clinical symptoms in adults with attention-deficit/hyperactivity disorder. Neurosci Lett 469:102-106

Vernon DJ (2005) Can neurofeedback training enhance performance? An evaluation of the evidence with implications for future research. Appl Psychophysiol Biofeedback 30:347-364

Wigal SB, Biederman J, Swanson JM, Yang R, Greenhill LL (2006) Efficacy and safety of modafinil film-coated tablets in children and adolescents with or without prior stimulant treatment for attention-deficit/hyperactivity disorder: pooled analysis of 3 randomized, double-blind, placebo-controlled studies. Prim Care Companion J Clin Psychiatry 8:352-360 